\section{Endovascular management of a refractory traumatic aneurysm of the internal carotid artery using a covered stent graft}

Sir,

Traumatic aneurysms of the internal carotid artery (ICA) although uncommon can be life-threatening and difficult to treat. Endovascular obliteration using detachable coils which has proved to be very effective in the management of cerebral aneurysms has been extended to manage traumatic aneurysms too. However, recanalization of these aneurysms has been a troublesome complication faced by interventional neuroradiologists. ${ }^{[1]}$

A 21-year-old male presented with repeated attacks of epistaxis following a road traffic accident. A cerebral angiogram revealed a traumatic aneurysm of the intracavernous segment of the left ICA. Patient underwent endovascular obliteration of the aneurysm using the Guglielmi Detachable Coils (GDC) three times. Each time angiographic obliteration of the aneurysm was confirmed but the patient would report back with epistaxis. Angiography demonstrated recanalization of the aneurysm each time. The patient reported to our institution with epistaxis two months after the third attempt at coiling the aneurysm.

Under endotracheal general anesthesia bilateral femoral access was obtained. Right ICA angiogram with a left carotid cross compression failed to show any cross flow to the left hemisphere. Left ICA angiogram revealed a small proximal filling of the $\mathrm{C} 2$ cavernous aneurysm [Figure 1]. Previous coil mass was noted. Over an exchange Teflon wire, a $7 \mathrm{~F}$ long sheath was placed in the left ICA. A 6F Envoy guiding catheter was placed in the left ICA at the skull base. Microcatheter was then navigated into the left middle cerebral artery and was exchanged for stabilizer exchange length wire.
Jomed Graftmaster stent graft was then deployed into the left ICA covering the neck of the aneurysm and two inflations were done at 14atm and 15atm respectively [Figure 2]. Post stenting left ICA angiogram showed good patency and anterograde flow of the left ICA and its subsequent branches. The ophthalmic artery however, was not visualized. There was no opacification of the aneurysm [Figure 3]. The patient was extubated and was placed on low molecular weight heparin (Enoxaparin $40 \mathrm{mg}$ twice daily for two days) along with aspirin and clopidogrel. Preoperatively the patient had received $300 \mathrm{mg}$ of Clopidogrel $6 \mathrm{~h}$ prior to the surgery as a bolus. The postoperative stay was uneventful. The patient had no focal neurological deficit and suffered no further episodes of epistaxis. Aspirin and Clopidogrel were continued for one year. A follow-up angiogram after one year showed patency of the graft with no opacification of the aneurysm [Figure 4]. Clopidogrel was then discontinued and the patient was continued on Aspirin.

Traumatic aneurysms (TA) of the ICA are rare and constitute only $0.15-0.04 \%$ of all intracranial aneurysms. Many of the TAs of the cavernous portion of the ICA present with carotido cavernous fistula (CCF) on rupture. Otherwise they may present with epistaxis or sphenoid sinus aneurysm. TA of the ICA may coexist with a CCF and may be recognized once the CCF is occluded. Angiographic features of a TA include delayed filling and emptying of the sac and an irregular contour without a visible neck at times. However, history of recent trauma and its relationship to the location of TA remain the main criteria for diagnosis. ${ }^{[2]}$ Endovascular embolization using GDC is reported as a safe and effective treatment for TA. However, in the acute phase, placement of GDC in the aneurysm may present an extreme danger of aneurysmal rupture due to the fragility of the aneurysm and lack of a formal wall or neck. ${ }^{[2]}$ Recanalization of aneurysm with recurrence has been seen in 10-20\% of cases after GDC embolization. ${ }^{[1]}$ Hence more innovative techniques have been evolved by interventional neuroradiologists. Covered stent grafts have also been used to exclude the aneurysm from the circulation. ${ }^{[1,3]}$ The deployment of a covered stent across the neck of the aneurysm can result in immediate obliteration of the aneurysm, thus affording an attractive solution to this potentially dangerous condition. The authors have used a Jomed coronary stent graft which has a sandwich-like design to fix a thin polytetrafluoroethylene (PTFE) membrane between two stainless steel stents. This design was modified to extend the membrane up to the stent edges. Saatchi et al., have used Jomed stents for the endovascular treatment of 25 ICA aneurysms located on the petrous, cavernous and ophthalmic segments. In their series occlusion of the ophthalmic artery was seen in eight cases. As in our patient, none of the 


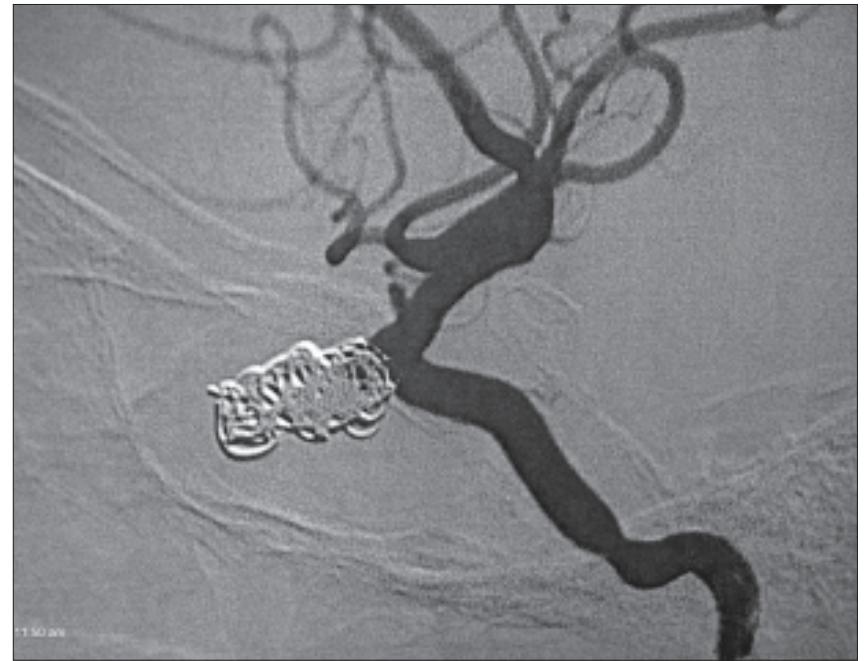

Figure 1: Left ICA angiogram showing the coils in situ with partial refilling of the aneurysm

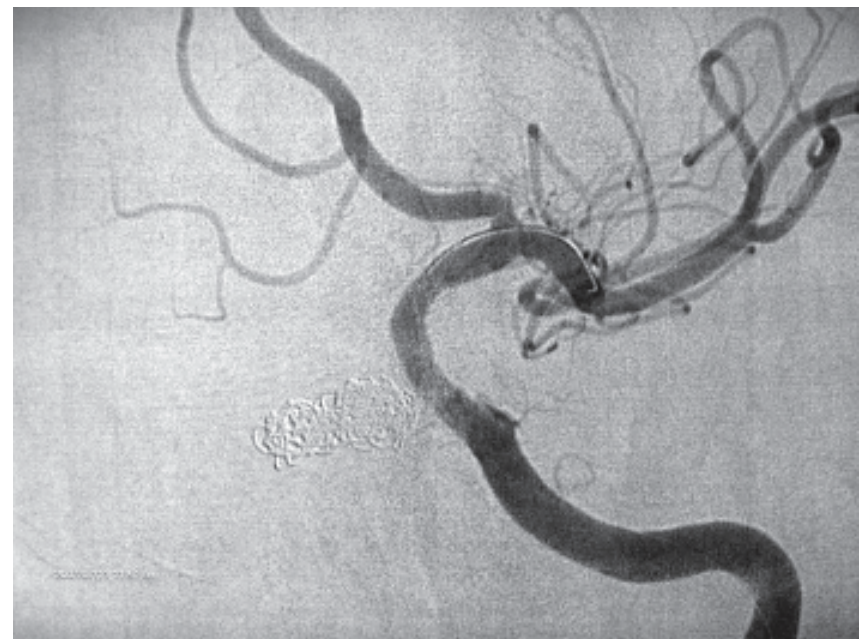

Figure 2: Left ICA angiogram after stent placement and first balloon inflation

eight cases of ophthalmic artery occlusion resulted in visual dysfunction. However, they recommend extreme caution to prevent accidental closure of the anterior choroidal artery. ${ }^{[1]}$

However, one of the main problems encountered in stent usage is the difficulty in navigating the stent through the tortuous intracranial vessels which may result in dissection and vasospasm of the cerebral arteries. The authors were able to use the $7 \mathrm{~F}$ long sheath, 6F Envoy combination for placement of the stent graft. The young age of the patient might have also contributed in the easy and uneventful passage of the Jomed stent. Hua Li et al., have described their experience in the management of the pseudoaneurysms in the intracranial segment of the ICA with covered stents specially designed for use in the intracranial vasculature. ${ }^{[3]}$ Their stents had staggered single linking points with a strut thickness of only $0.06 \mathrm{~mm}$ and consisted of only one stent and one piece of PTFE membrane stuck together

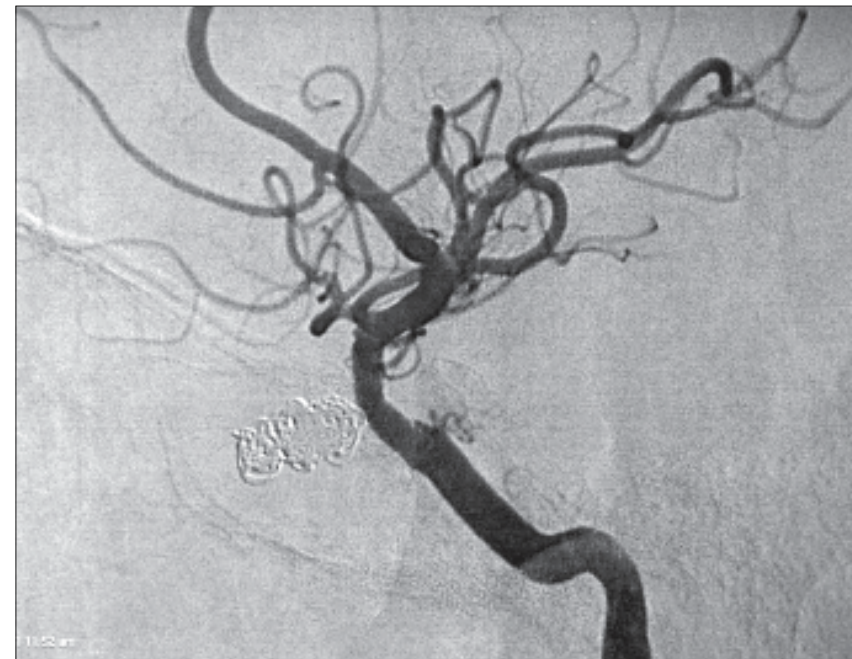

Figure 3: Left ICA angiogram after stent deployment and two balloon inflations showing obliteration of the aneurysm

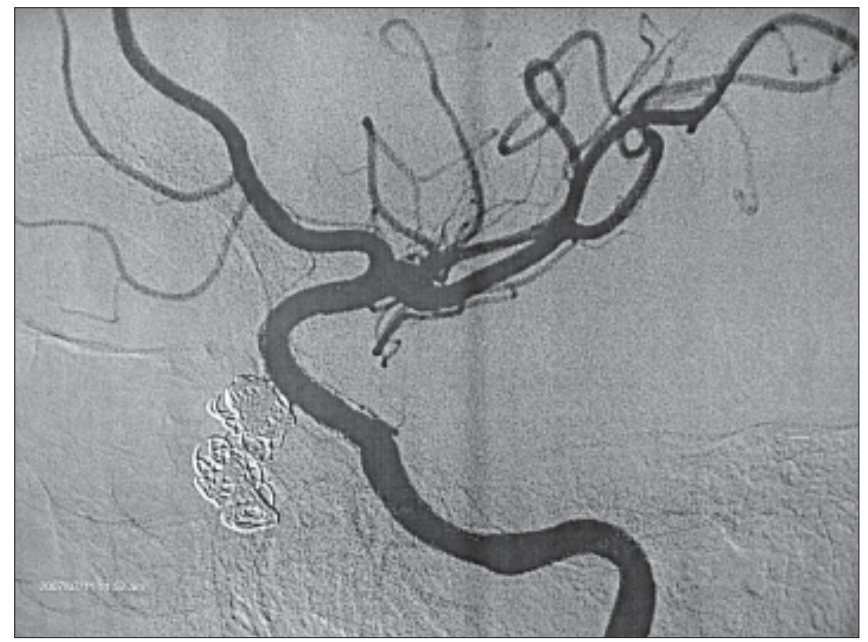

Figure 4: Left ICA angiogram after one year confirming obliteration of the aneurysm with no recanalization or stent stenosis

thus making the stent extremely flexible. ${ }^{[3]}$

The use of a covered stent in the obliteration of an aneurysm of the petrous, cavernous and ophthalmic portions of the ICA is not only effective but also safe. ${ }^{[4,5]}$ It can prove as a more effective alternative to GDC embolization in refractory cases.

\section{Balaji S. Pai, Uday Limaye ${ }^{1}$, Ravi G. Varma}

Department of Neurosurgery, M. S. Ramaiah Medical College, Bangalore, India, ${ }^{1}$ Department of Interventional Neuroradiology, King Edward Memorial Hospital, Mumbai, India. E-mail: balajipai65@yahoo.co.in

\section{References}

1. Saatci I, Cekirge HS, Ozturk MH, Arat A, Ergungor F, Sekerci Z, et al. Treatment of internal carotid artery aneurysms with a covered stent: experience in 24 patients with mid-term follow-up results. AJNR Am J Neuroradiol 2004;25:1742-9.

2. Luo CB, Teng MM, Chang FC, Lirng JF, Chang CY. Endovascular management of the traumatic cerebral aneurysms associated with 
traumatic carotid cavernous fistulas. AJNR Am J Neuroradiol 2004;25:501-5.

3. Li MH, Gao BL, Wang YL, Fang C, Li YD. Management of pseudoaneurysms in the intracranial segment of the internal carotid artery with covered stents specially designed for use in the intracranial vasculature: technical notes. Neuroradiology 2006;48:841-6.

4. Maras D, Lioupis C, Magoufis G, Tsamopoulos M, Moulakakis K, Andrikopoulos V. Covered stent graft treatment of traumatic internal carotid artery pseudoaneurysms: A review. Cardiovase Intervent Radiol 2006;29:958-68.

5. Yi AC, Palmer E, Luh GY, Jacobson JP, Smith DC. Endovascular treatment of carotid and vertebral pseudoaneurysms with covered stents. AJNR Am J Neuroradiol 2008;29:983-7.

Accepted Date: 30-06-2008 